\title{
UNDER THE DEGREE OF SOME FINITE LINEAR GROUPS
}

\author{
BY \\ HARVEY I. BLAU( $\left.{ }^{1}\right)$
}

\begin{abstract}
Let $G$ be a finite group with a cyclic Sylow $p$-subgroup $P$ for some prime $p \geqq 13$. Assume that $G$ is not of type $L_{2}(p)$, and that $G$ has a faithful indecomposable modular representation of degree $d \leqq p$. This paper offers several improvements of the known bound $d \geqq(7 p) / 10-1 / 2$. In particular, $d \geqq 3(p-1) / 4$. Other bounds are given relative to the order of the center of $G$ and the index of the centralizer of $P$ in its normalizer.
\end{abstract}

1. Introduction. A finite group is of type $L_{2}(p)$ if each of its composition factors is either a $p$-group, a $p^{\prime}$-group or isomorphic to $\operatorname{PSL}(2, p)$. Feit [5] proved

THEOREM 1. Let $G$ be a finite group with a cyclic $S_{p}$-subgroup $P$ for some prime $p$. Assume that $G$ is not of type $L_{2}(p)$. Suppose that there is a faithful indecomposable $K G$-module $L$ of dimension $d \leqq p$, where $K$ is a field of characteristic $p$. Then $p \neq 2$, $|P|=p,\left.L\right|_{P}$ is indecomposable, and $\mathscr{C}_{G}(P)=P \times \mathscr{Z}(G)$. Furthermore $d \geqq 2(p-1) / 3$ and $d \geqq(7 p) / 10-\frac{1}{2}$ in case $p \geqq 13$.

If $p<13$, all relevant groups with faithful indecomposable $K G$-modules of dimension less than $p-2$ are known, including the Janko group of degree 7 where $p=11$ [11]. The question of whether there exist any groups satisfying Theorem 1 with $p \geqq 13$ and $d<p-2$ remains open. Should any occur, they would lead to new simple groups.

This paper offers several improvements of the lower bound $(7 p) / 10-\frac{1}{2}$ for the dimension of $L$ when $p \geqq 13$. We easily show $d \geqq 3(p-1) / 4$ (Theorem 5.7). If $d=3(p-1) / 4$ then $L$ is self-dual, $|\mathscr{Z}(G)|=2$, and $\left|\mathscr{N}_{G}(P): \mathscr{C}_{G}(P)\right|=(p-1) / 2$ (Theorem 6.4). Other theorems relate $d$ to $|\mathscr{Z}(G)|=z$ and $\left|\mathscr{N}_{G}(P): \mathscr{C}_{G}(P)\right|=e$ $=(p-1) / t$. In particular, if $e$ is even and $z$ odd then $d$ is either odd or equal to $p-1$ (Theorem 5.12). $d \geqq p-(e / 2+1)$ if $e$ is even, and $d \geqq p-((e-1) / 2+t)$ if $e$ is odd (Theorem 7.1). This last result serves to improve an inequality due to Brauer [3] for groups with a complex representation of degree less than $p-1$. (See the remarks in §7.)

Received by the editors May 18, 1970.

AMS 1969 subject classifications. Primary 2025, 2080; Secondary 2075.

Key words and phrases. Indecomposable modular representation, small degree, cyclic Sylow $p$-subgroup, symmetric decomposition, skew decomposition, irreducible complex representation.

( $\left.{ }^{1}\right)$ Most of the results in this paper were part of the author's doctoral dissertation, written under the direction of Walter Feit and submitted to Yale University. This research was partially supported by the Army Research Office (Durham).

Copyright (C) 1971, American Mathematical Society 
The methods of [5] are exploited, beginning with a generalized local theory in $\$ 2$. The result in $\$ 3$ on symmetric and skew decomposition is used in several proofs in the sequel. A theorem of Feit on invariants and the Green correspondence is combined with the structure theory of a block with cyclic defect group in $\$ 4$, providing some useful information. The main results are established in $\$ \S 5,6,7$. Finally, a table is given for the possible values of $d$ when $13 \leqq p \leqq 31$.

$G$ denotes a finite group, $K$ a field, $P$ a $S_{p}$-subgroup of $G, N=\mathscr{N}_{G}(P)$ and $C=\mathscr{C}_{G}(P)$. If $M$ and $W$ are $K G$-modules, $M+W$ means their direct sum and $M^{*}$ is the dual of $M$. Further notation and terminology are either standard or explained en route.

2. Local theory. A mild generalization of results of Thompson [14] and Feit [5] is presented. The proofs in these sources for the prototypes of Lemmas 2.1-2.6 below carry over virtually unchanged, so we omit those proofs here.

In this section, $P$ is a cyclic group of order $q=p^{n}$ for some fixed prime $p$, and $P \triangleleft P H$ where $H$ is an abelian $p^{\prime}$-group. Let $K$ be a field of characteristic $p$ which is a splitting field for $H$, so that the $|H|$ irreducible (linear) characters of $H$ are afforded by $K$-representations. Let char $H=\left\{\lambda_{i}\right\}$ be the set of all such characters.

LEMMA 2.1. For each integer $s$ with $1 \leqq s \leqq q$ and each $\lambda \in$ char $H$, there is an indecomposable KPH-module $V_{s}(\lambda)$ such that $\operatorname{dim}_{K} V_{s}(\lambda)=s,\left.V_{s}(\lambda)\right|_{P}$ is indecomposable, and if $U$ is the unique submodule of $V_{s}(\lambda)$ with $\operatorname{dim} U=1$, then $u h=\lambda(h) u$ for all $u \in U, h \in H$. Every indecomposable $K P H$-module is isomorphic to some $V_{s}(\lambda)$; $V_{s}(\lambda) \approx V_{t}(\mu)$ if and only if $s=t$ and $\lambda=\mu ; V_{s}(\lambda)$ is projective if and only if $s=q$. Furthermore for each $1 \leqq i \leqq s$, each $V_{s}(\lambda)$ has a unique submodule $U_{i}$ with $\operatorname{dim} U_{i}=i$; $U_{i} \approx V_{i}(\lambda)$.

Let $\alpha$ be the linear character: $H \rightarrow K$ given by

$$
h^{-1} y h=y^{\alpha(h)}, \quad \text { all } y \in P, h \in H .
$$

Then $\alpha(H) \subseteq F-\{0\}$, where $F$ is the prime subfield of $K$. In the sequel, $V_{s}(\lambda)$ is defined as in Lemma 2.1. Set $V_{0}(\lambda)=0$ for all $\lambda \in$ char $H$. If $H=\langle 1\rangle$, set $V_{s}(\lambda)=V_{s}$. If $h \in H$, $\operatorname{det}_{s}(\lambda)(h)$ means the determinant of $h$ acting as a linear transformation on $V_{s}(\lambda)$.

Lemma 2.2. Let $V_{i}(\lambda) \approx U_{i} \subseteq V_{s}(\lambda)$ for $0 \leqq i \leqq s$. Then $V_{s}(\lambda) / U_{i} \approx V_{s-i}\left(\lambda \alpha^{-i}\right)$.

As a corollary there is

Lemma 2.3. $V_{s}(\lambda)^{*} \approx V_{s}\left(\lambda^{-1} \alpha^{s-1}\right)$ and $\operatorname{det}_{s}(\lambda)(h)=\lambda^{s} \alpha^{-s(s-1) / 2}(h)$ for all $h \in H$.

Lemma 2.4. Assume $|P|=p$. If $1 \leqq s \leqq t$ and $s+t \leqq p$, then

$$
V_{s}(\lambda) \otimes V_{t}(\mu) \approx \sum_{i=0}^{s-1} V_{s+t-1-2 i}\left(\lambda \mu \alpha^{-i}\right)
$$

Lemma 2.5. Assume $|P|=p . V_{s}(\lambda) \otimes V_{p}(\mu) \approx \sum_{i=0}^{s-1} V_{p}\left(\lambda \mu \alpha^{-i}\right)$ for $1 \leqq s \leqq p$. 
Lemma 2.6. Assume $|P|=p$. If $1 \leqq b \leqq c$ and $b+c \leqq p$, then

$$
V_{p-b}(\beta) \otimes V_{c}(\gamma) \approx \sum_{i=0}^{b-1} V_{p-b-c+1+2 i}\left(\beta \gamma \alpha^{-c+1+i}\right)+\sum_{j=0}^{c-b-1} V_{p}\left(\beta \gamma \alpha^{-j}\right) .
$$

The following lemma is proved with a technique due to Green [10] and employed by Feit in [5].

LEMMA 2.7. If $M \approx V_{q}(\pi)+V_{t}(\tau), V_{s}(\sigma) \approx S \subseteq M$ and $M / S \approx V_{r}(\rho)$ where $t, r, s<q$, then $\sigma=\pi$ and $\rho=\tau=\pi \alpha^{r-1}$.

Proof. First observe that in any direct sum $V_{j}(\mu)+V_{i}(\lambda)$, the elements fixed by $P$ form a space of $K$-dimension two. Hence any submodule is a direct sum of at most two indecomposable summands. Since $s+r=q+t$ where $r<q$, then $s>t$. Thus, $S \cap V_{q}(\pi) \neq\{0\}$, hence $S$ and $V_{q}(\pi)$ share a one-dimensional $K P H$-module, so $\sigma=\pi$. Also $S \cap V_{1}(\tau)=\{0\}$ implies the image of $V_{1}(\tau)$ is nonzero in $M / S$, hence $\rho=\tau$. Observe

$$
\begin{aligned}
& V_{q}\left(\rho \alpha^{1-r}\right) / V_{q-r}\left(\rho \alpha^{1-r}\right) \approx V_{r}(\rho) \text { and } \\
& \left(V_{q}(\pi)+V_{q}\left(\tau \alpha^{1-t}\right)\right) / X \approx V_{q}(\pi)+V_{t}(\tau), \text { where } X \approx V_{q-t}\left(\tau \alpha^{1-t}\right) .
\end{aligned}
$$

Hence there exists $W$ with $\left(V_{q}(\pi)+V_{q}\left(\tau \alpha^{1-t}\right)\right) \supseteq W \supseteq X, W / X \approx S$ and

$$
\left(V_{q}(\pi)+V_{q}\left(\tau \alpha^{1-t}\right)\right) / W \approx V_{r}(\rho) .
$$

Combining (2.8) and (2.9) with Schanuel's theorem [10, (1.6e)] gives

$$
W+V_{q}\left(\rho \alpha^{1-r}\right) \approx V_{q-r}\left(\rho \alpha^{1-r}\right)+V_{q}(\pi)+V_{q}\left(\tau \alpha^{1-t}\right) .
$$

Thus $W$ has a projective summand and $W, X$ satisfy hypotheses similar to those on $M, S$ so $V_{q}\left(\tau \alpha^{1-t}\right) \subseteq W$. Then by $(2.10)$ and the Krull-Schmidt theorem, $V_{q}\left(\rho \alpha^{1-r}\right) \approx V_{q}(\pi)$. Hence $\rho=\pi \alpha^{r-1}$.

Definition. If $U \approx V_{1}(\lambda)$ is an irreducible constituent of a $K P H$-module $M$, then we say that $\lambda$ is an $H$-value of $M$. This is equivalent to $\lambda(h)$ being an eigenvalue of $h$ acting on $M$, for all $h \in H$. $\lambda$ is a main $H$-value (mv) if there is $x \neq 0$ in $M$ with $x h=\lambda(h) x$ and $x y=x$ for all $h \in H, y \in P$.

The $H$-values of $V_{s}(\lambda)$ are $\lambda, \lambda \alpha^{-1}, \ldots, \lambda \alpha^{-s+1}$ by Lemma 2.2, and the unique $\mathrm{mv}$ of $V_{s}(\lambda)$ is $\lambda$. Considering the set of elements fixed by $P$ immediately gives

Proposition 2.11. Let $M=\sum_{i=1}^{n} V_{d_{i}}\left(\lambda_{i}\right)$ where $1 \leqq d_{i} \leqq q$ and $\lambda_{i} \in$ char $H$ for $1 \leqq i \leqq n$. The $H$-values of $M$ are $\bigcup_{i=1}^{n}\left\{\lambda_{i}, \lambda_{i} \alpha^{-1}, \ldots, \lambda_{i} \alpha^{-d_{i}+1}\right\}$ and the mv's are the $\lambda_{i}$.

The $H$-values of $V_{d_{i}}\left(\lambda_{i}\right)$ are called projective $H$-values (pv) (resp. nonprojective $H$-values (npv)), and $\lambda_{i}$ is projective main value (pmv) (resp. nonprojective main value (npmv)) if $d_{i}=q$ (resp. $\left.d_{i}<q\right)$. Of course, a given $\lambda$ may be both a pv and a npv of $M$.

3. Symmetric and skew decomposition. If $K$ is a field of characteristic not equal to two, and $L$ a $K G$-module with $\operatorname{dim}_{K} L=d$, then $L \otimes L=A+B$, where $A$ is the 
subspace of symmetric tensors and $B$ the subspace of skew-symmetric tensors. $A$ and $B$ are $K G$-modules with $\operatorname{dim}_{K} A=d(d+1) / 2$ and $\operatorname{dim}_{K} B=d(d-1) / 2$. For any subgroup $H$ of $G,\left.A\right|_{H}$ and $\left.B\right|_{H}$ are the symmetric, resp. skew, summands of $\left.\left.L\right|_{H} \otimes L\right|_{H}$. If $\left\{x_{i}\right\}$ is a $K$-base for $L$, then

$$
A=\left\langle x_{i} \otimes x_{j}+x_{j} \otimes x_{i}\right\rangle, \quad B=\left\langle x_{i} \otimes x_{j}-x_{j} \otimes x_{i}\right\rangle .
$$

Suppose $\left\{x_{i}\right\}$ consists of eigenvectors with respective eigenvalues $\varepsilon_{i}$ for some $g \in G$. Then by (3.1), the eigenvalues of $g$ on $A$ consist exactly of $\left\{\varepsilon_{i}^{2}\right\}$ plus the eigenvalues of $g$ on $B$.

For the rest of this section, let field $K$ and group $P H$ satisfy the hypotheses of $\S 2$, with $|P|=p$. Then for any integer $d$ with $p / 2<d<p$, and $s=p-d$, Lemma 2.6 says, for any $\lambda \dot{\epsilon} \operatorname{char} H$,

$$
V_{d}(\lambda) \otimes V_{d}(\lambda) \approx \sum_{i=0}^{s-1} V_{2 i+1}\left(\lambda^{2} \alpha^{s+i}\right)+\sum_{i=s}^{p-1-s} V_{p}\left(\lambda^{2} \alpha^{s+i}\right) .
$$

Lemma 3.3. Let $s=p-d$, where $p / 2<d<p$. Let $A+B$ be the decomposition of $V_{d}(\lambda) \otimes V_{d}(\lambda)$ into symmetric and skew parts. Then $A$ is the direct sum of exactly those summands in (3.2) (projective and nonprojective) with $i \equiv s(\bmod 2) . B$ is the direct sum of the summands in (3.2) with $i \equiv s-1(\bmod 2)$.

Proof. By the Krull-Schmidt theorem, the summands of (3.2) are distributed between $A$ and $B$. The remarks above and Lemma 2.2 show that

$$
(H \text {-values of } A)=\left\{\lambda^{2},\left(\lambda \alpha^{-1}\right)^{2}, \ldots,\left(\lambda \alpha^{-d+1}\right)^{2}\right\} \cup(H \text {-values of } B) \text {. }
$$

Assume first that $P H$ is a Frobenius group with Frobenius kernel $P$ (so that $\alpha$ is faithful on $H$ and char $H=\langle\alpha\rangle$ ), and that $|H|=p-1$. Since $|H|$ is even, it makes sense to distinguish between even and odd powers of $\alpha$. Since $d>p / 2,\left\{\lambda^{2},\left(\lambda \alpha^{-1}\right)^{2}\right.$, $\left.\ldots,\left(\lambda \alpha^{-d+1}\right)^{2}\right\}$ covers each even power at least once, and $|H|=p-1$ implies

$$
\left\{\lambda^{2} \alpha^{s \pm i} \mid 0 \leqq i \leqq s-1\right\} \cap\left\{\lambda^{2} \alpha^{s+i} \mid s \leqq i \leqq p-s-1\right\}=\varnothing
$$

The two sets given in (3.5) are the $H$-values of the nonprojective summands and the mv's of the projective summands, respectively. Each $\gamma \in$ char $H$ is an $H$-value of $V_{p}(\pi)$ twice if $\gamma=\pi$, but exactly once if $\gamma \neq \pi$.

Of the $p-2 s$ projective summands, suppose more lie in $B$ than in $A$. Then each of $\lambda^{2} \alpha^{s+i}, s \leqq i \leqq p-s-1$, occurs more times in $B$ than in $A$ as an $H$-value which is not a mv, and the majority of them occur additionally in $B$ as pmv's. (3.5) shows they are not balanced by nonprojective $H$-values, and this contradicts (3.4). So more projective summands lie in $A$ than in $B$.

Now $\lambda^{2} \alpha^{2 s-1}$ is an $H$-value of only $V_{2 s-1}\left(\lambda^{2} \alpha^{2 s-1}\right)$ among the nonprojective summands, and is a non-mv just once for each $V_{p}\left(\lambda^{2} \alpha^{s+i}\right), s \leqq i \leqq p-s-1$. So if $V_{2 s-1}\left(\lambda^{2} \alpha^{2 s-1}\right) \subseteq A,(3.4)$ implies there is one more projective summand in $B$ than in $A$ in order that the odd powers of $\alpha$ balance as $H$-values, a contradiction. Hence, 
$V_{2 s-1}\left(\lambda^{2} \alpha^{2 s-1}\right) \subseteq B$. To balance $\lambda^{2} \alpha^{2 s-1}$, there is exactly one more projective summand in $A$.

Similarly, $\lambda^{2} \alpha^{2 s-2}$ is an $H$-value of only $V_{2 s-1}\left(\lambda^{2} \alpha^{2 s-1}\right), V_{2 s-3}\left(\lambda^{2} \alpha^{2 s-2}\right)$ and each projective. $V_{2 s-1}\left(\lambda^{2} \alpha^{2 s-1}\right)$ with the projectives leaves $\lambda^{2} \alpha^{2 s-2}$ balanced between $A$ and $B$. This is an even power of $\alpha$, so (3.4) implies $V_{2 s-3}\left(\lambda^{2} \alpha^{2 s-2}\right) \subseteq A$.

Consider $V_{2 j+1}\left(\lambda^{2} \alpha^{s+j}\right)$ for $0 \leqq j<s-2$, and suppose for all $j<k \leqq s-1$,

$$
\begin{aligned}
& V_{2 k+1}\left(\lambda^{2} \alpha^{s+k}\right) \subseteq B \quad \text { if } k \equiv s-1 \quad(\bmod 2), \\
& \subseteq A \text { if } k \equiv s \quad(\bmod 2) \text {. }
\end{aligned}
$$

$\lambda^{2} \alpha^{s+j}$ is an $H$-value of only $V_{2 j+1}\left(\lambda^{2} \alpha^{s+j}\right), V_{2 k+1}\left(\lambda^{2} \alpha^{s+k}\right)$ for each $k>j$, and of each projective. If $j \equiv s-1(\bmod 2)$ then our inductive assumption implies the $V_{2 k+1}\left(\lambda^{2} \alpha^{s+k}\right)$ and the projectives give an extra $\lambda^{2} \alpha^{s+j}$ as an $H$-value to $A$. So (3.4) implies $V_{2 j+1}\left(\lambda^{2} \alpha^{s+j}\right) \subseteq B$. If $j \equiv s(\bmod 2)$, our assumption says that the $V_{2 k+1}\left(\lambda^{2} \alpha^{s+k}\right)$ and the projectives distribute $\lambda^{2} \alpha^{s+j}$ evenly between $A$ and $B$. Hence, $V_{2 j+1}\left(\lambda^{2} \alpha^{s+j}\right) \subseteq A$. Induction downwards shows

$$
\begin{aligned}
& A=\sum_{0 \leqq i \leqq s-1: i \leqq s(\bmod 2)} V_{2 i+1}\left(\lambda^{2} \alpha^{s+i}\right)+(p-2 s+1) / 2 \text { projectives, } \\
& B=\sum_{0 \leqq i \leqq s-1 ; i \leqq s-1(\bmod 2)} V_{2 i+1}\left(\lambda^{2} \alpha^{s+i}\right)+(p-2 s-1) / 2 \text { projectives. }
\end{aligned}
$$

For any group $P$ of odd prime order $p$, the Frobenius group $P H$ as above may be constructed. By restriction to $P$, our results imply that if $V_{d} \otimes V_{d}=A^{\prime}+B^{\prime}$, decomposition into symmetric and skew parts, then

$$
\begin{aligned}
& A^{\prime}=\sum_{0 \leqq i \leqq s-1 ; i \leqq s(\bmod 2)} V_{2 i+1}+((p-2 s+1) / 2) V_{p}, \\
& B^{\prime}=\sum_{0 \leqq i \leqq s-1 ; i \leqq s-1(\bmod 2)} V_{2 i+1}+((p-2 s-1) / 2) V_{p} .
\end{aligned}
$$

Now make no special assumption about $P H . \quad V_{d}(\lambda) \otimes V_{d}(\lambda)$ contains a unique indecomposable summand of dimension $2 i+1$, for each $i$ with $0 \leqq i \leqq s-1$. So restricting to $P$ and applying (3.7) shows that (3.6) remains true. Finally, it now follows that the projective summands distribute between $A$ and $B$ as in the statement of this lemma in order that (3.4) be satisfied.

In the same way (and with less trouble), one obtains the following results: If $2 s \leqq p$, then Lemma 2.4 says $V_{s}(\lambda) \otimes V_{s}(\lambda) \approx \sum_{i=0}^{s-1} V_{2 i+1}\left(\lambda^{2} \alpha^{1-s+i}\right)$.

Lemma 3.8. Let $2 s \leqq p \neq 2$. Let $A+B$ be the decomposition of $V_{s}(\lambda) \otimes V_{s}(\lambda)$ into symmetric and skew parts. Then $A$ is the direct sum of the $V_{2 i+1}\left(\lambda^{2} \alpha^{1-s+i}\right)$ with $i \equiv s-1(\bmod 2), 0 \leqq i \leqq s-1 ; B$ is the direct sum of the $V_{2 i+1}\left(\lambda^{2} \alpha^{1-s+i}\right)$ with $i \equiv s(\bmod 2), 0 \leqq i \leqq s-2$.

4. Blocks and the Green correspondence. Here is a special case of the Green correspondence (see Thompson [14]): Let $K$ be a field of characteristic $p$ and $G$ a 
finite group with a $S_{p}$-subgroup $P$ which is a T.I. set. There is a one-to-one correspondence between all nonprojective indecomposable $K G$-modules $X$, and all nonprojective indecomposable $K N$-modules $V: X \leftrightarrow V$ if and only if $V$ is the unique nonprojective indecomposable summand of $\left.X\right|_{N}$, or equivalently, $X$ is the unique nonprojective summand of $V^{G}$. If $X \leftrightarrow V$, then $X^{*} \leftrightarrow V^{*}$.

A nonzero element $x$ of a $K G$-module is called an invariant if $x g=x$, all $g \in G$.

THEOREM 4.1 (FEIT). If $X \leftrightarrow V$ as above, then $X$ has invariants if and only if $V$ has invariants.

Proof. Let $Q_{0}$ be the projective indecomposable $K G$-module whose socle is the trivial one-dimensional $K G$-module. $Q_{0}$ is, of course, a direct summand of $K G$ with socle $K\left(\sum_{g \in G} g\right)$. If $X$ contains a nonzero element of the form $\sum_{g \in G} y g$ for some $y \in X$, then the map $f: K G \rightarrow X$ defined by $f\left(\sum_{g \in G} a_{g} g\right)=\sum_{g \in G} a_{g} y g$, where $a_{g} \in K$, induces a $K G$-isomorphism of $Q_{0}$ into $X$, a contradiction. Thus, $N_{G,\langle 1\rangle}(X)=0$, so that $H^{0}(G,\langle 1\rangle, X)=\operatorname{Inv}_{G}(X)$ (see [7, II.3]). Similarly, $H^{0}(N,\langle 1\rangle, V)=\operatorname{Inv}_{N}(V)$. Since $H^{0}(G,\langle 1\rangle, X) \approx H^{0}(N,\langle 1\rangle, V)$ [7, III.5.9], the theorem follows.

Now let $|P|=p$, let $\mathcal{O}$ be the ring of integers in a $p$-adic number field $k, \mathscr{P}$ the maximal ideal of $\mathcal{O}, K=\mathcal{O} / \mathscr{P}$, and assume all irreducible $k G$ and $K G$ modules are absolutely irreducible. $B$ will denote a $p$-block of defect 1 .

Each block $B$ is associated with a tree (see Brauer [1], Dade [4], Rothschild [13]). Say that the graph of $B$ has $e$ edges, corresponding to irreducible modular characters (and to their corresponding projective indecomposables), and hence $e+1$ vertices, corresponding to $p$-conjugate families of ordinary irreducible characters. In only one family, said to lie on the exceptional vertex, is there more than one character (there are $(p-1) / e$ ). If $e=p-1$, we pick the "exceptional" vertex arbitrarily.

Definition. If $M$ is an irreducible $K G$-module in $B$, the remainder of $M$ (rem $M$ ) is the unique integer $m$ with $1 \leqq m<p$ and $\operatorname{dim}_{K} M \equiv m(\bmod p)$. The separation of $M(\operatorname{sep} M)$ is the number of vertices the edge $M$ separates from the exceptional. (If $e=p-1$, sep $M$ depends on our choice of the exceptional vertex.)

An argument of Rothschild shows there is an integer $r \not \equiv 0(\bmod p)$ with

$$
r(\operatorname{sep} M) \equiv \pm \operatorname{rem} M(\bmod p)
$$

where the result alternates along paths to the exceptional. As a consequence, all remainders in $B$ are congruent $(\bmod p)$ to elements of

$$
\{r, 2 r, \ldots, e r\} \cup\{-e r,-(e-1) r, \ldots,-r\} .
$$

Results of Brauer [2, I] give

$$
\text { If } N / P \text { is abelian, then } e=|N: C| \text { and } r=1 \text {. }
$$

The correspondence $L \rightarrow L^{*}$, where $L$ is an irreducible $k G$ - or $K G$-module, gives an incidence preserving map of the tree for $B$ to the tree for a block $B^{\prime}$. If $B$ contains an ordinary or modular irreducible which is isomorphic to its dual (Brauer 
[1, Theorem 13] shows that the latter implies the former), then the map sends $B$ to itself. The same theorem of Brauer implies that those modular (resp. ordinary) irreducibles equal to their duals lie on the edges (resp. vertices) of a single real stem across which the map $L \rightarrow L^{*}$ reflects the tree. The exceptional vertex in such a block, if $e<p-1$, must also lie on the stem. (This discussion is given by Tuan [15] in the case of the principal block.)

For the rest of $\S 4$, assume $N=P H$ where $H$ is an abelian $p^{\prime}$-group. Then (4.3) and $\S \S 2,3$ apply. $\lambda$ is called an $H$-value of a $K G$-module $L$ if and only if it in $H$-value of $\left.L\right|_{N}$.

Proposition 4.4. For any $\lambda \in$ char $H$, there is at most one irreducible KG-module $X$ such that $X \leftrightarrow V_{t}(\lambda)$ by the Green correspondence for some positive integer $t<p$.

Proof. Suppose $X$ and $Y$ are distinct irreducibles with $X \leftrightarrow V_{t}(\lambda)$ and $Y \leftrightarrow V_{s}(\lambda)$ $\operatorname{Hom}_{K G}(X, Y)=0=\operatorname{Hom}_{K G}(Y, X)$, so by Theorem 4.1 , there are no invariants in the nonprojective summands of $V_{t}(\lambda)^{*} \otimes V_{s}(\lambda)$ or of

$$
V_{s}(\lambda)^{*} \otimes V_{t}(\lambda)=V_{s}\left(\lambda^{-1} \alpha^{s-1}\right) \otimes V_{t}(\lambda) \text {. }
$$

Without loss, assume $s \leqq t$. If $s+t \leqq p$,

$$
V_{s}\left(\lambda^{-1} \alpha^{s-1}\right) \otimes V_{t}(\lambda) \approx \sum_{i=0}^{s-1} V_{s+t-1-2 i}\left(\lambda^{-1} \alpha^{s-1} \lambda \alpha^{-i}\right)=\sum_{i=0}^{s-1} V_{s+t-1-2 i}\left(\alpha^{s-1-i}\right),
$$

by Lemma 2.4. But one of the npmv's is $\alpha^{0}=1$, a contradiction. If $s+t>p$, let $t=p-b$. Then $b<s$ and

$$
V_{s}\left(\lambda^{-1} \alpha^{s-1}\right) \otimes V_{p-b}(\lambda) \approx \sum_{i=0}^{b-1} V_{p-b-s+1+2 i}\left(\lambda^{-1} \alpha^{s-1} \lambda \alpha^{1-s+i}\right)+(\text { projectives })
$$

by Lemma 2.6 , and $\alpha^{0}$ is a npmv, a contradiction.

The following result is proven by Feit (not yet published) in a more general setting.

Proposition 4.5. Let $M, W$ and $R$ be nonprojective indecomposable $K G$-modules with $M \leftrightarrow V_{m}(\mu), W \leftrightarrow V_{w}(\gamma), R \leftrightarrow V_{r}(\rho), M \subseteq W$ and $W / M \approx R$. Then

(a) $m+r<p$ implies $m+r=w$ and $\rho=\mu \alpha^{-m}, \gamma=\mu$;

(b) $m+r>p$ implies $m+r=p+w$ and $\rho=\gamma=\mu \alpha^{r-1}$.

Furthermore, if one of $R$ or $M$ is irreducible, then it is uniquely determined by the other and one of conditions (a) or (b).

Proof. Treat the problem locally. All projective summands of $\left.M\right|_{N}$ appear in $\left.W\right|_{N}$, and in addition so do those of $\left.R\right|_{N}$. Factor out the former, split off the latter, and hence assume $\left.M\right|_{N}=V_{m}(\mu),\left.R\right|_{N}=V_{r}(\rho)$, so $\operatorname{dim}_{K} W=m+r$. If $m+r<p$, then $\left.W\right|_{N}=V_{w}(\gamma)+$ no projectives, with $m+r=w . V_{m+r}(\gamma) / V_{m}(\mu) \approx V_{r}(\rho)$ implies $\gamma=\mu$ and $\rho=\mu \alpha^{-m}$ by Lemma 2.2. If $m+r>p$, then $\left.W\right|_{N}=V_{w}(\gamma)+V_{p}(\pi)$, and we apply Lemma 2.7 to obtain $\pi=\mu$ and $\rho=\gamma=\mu \alpha^{r-1}$. 
Suppose $R$ is irreducible. If $m+r<p$, then $\rho=\mu \alpha^{-m}$, which depends only on $M$, and which by Proposition 4.4 determines $R$. If $m+r>p$, let $R=S^{*}$, so that where $S \leftrightarrow V_{r}(\sigma), V_{r}(\rho)=V_{r}(\sigma)^{*}=V_{r}\left(\sigma^{-1} \alpha^{r-1}\right)$ by Lemma 2.3. Then $\sigma^{-1} \alpha^{r-1}=\mu \alpha^{r-1}$, so $\sigma=\mu^{-1}$, and by Proposition 4.4 again, $S$ (hence $R \approx S^{*}$ ) is determined by $M$. If $M$ is irreducible, consider the dual $W^{*} \supseteq R^{*}$ and $W^{*} / R^{*} \approx M^{*}$.

Proposition 4.6. The npmv's of the indecomposable KG-modules in a single block $B$ of defect 1 all lie in a single coset of char $\left(H / \mathscr{C}_{H}(P)\right)$ in char $H$.

Proof. Let $U$ be the unique maximal submodule of an arbitrary projective indecomposable $K G$-module $Q$ in $B$. There is a chain of submodules $W=W_{0} \subseteq W_{1} \subseteq \cdots \subseteq W_{n}=U$ such that $W$ is the unique minimal submodule of $U$, hence all the $W_{i}$ are indecomposable, and the $W_{i} / W_{i-1}, 0 \leqq i \leqq n$, include all the distinct irreducible constituents of $Q$. Let $W_{i} / W_{i-1}$ have npmv $\lambda_{i}$ and $W_{i}$ have npmv $\gamma_{i}$. By Proposition 4.5, $\gamma_{i}$ is either $\gamma_{i-1}$ or $\lambda_{i}$, and $\lambda_{i}=\gamma_{i-1} \alpha^{j_{i}}$ for some integer $j_{i}$. Since $\left.\alpha\right|_{\mathscr{C}_{H}(P)}=1$, the $\lambda_{i}$ are all in a single coset of char $\left(H / \mathscr{C}_{H}(P)\right)$. Since projective indecomposables on adjacent edges of the tree have irreducible constituents in common, the proposition is true for all the modular irreducibles in $B$.

Let $X \leftrightarrow V_{s}(\sigma), 0<s<p$, be any indecomposable $K G$-module in $B$. Let $M$ be a maximal submodule of $X$. By induction on the dimension of $X$, we may assume that the npmv's of $M$ and of the irreducible constituents of $M$ are in the same coset of char $\left(H / \mathscr{C}_{H}(P)\right)$. We may also assume that $\sigma$ is not a npmv of $M,\left.M\right|_{N}$ $=\sum_{i} V_{m_{i}}\left(\mu_{i}\right)$ where each $m_{i}<p$, and $\operatorname{dim} X / M<p$. Now $\sigma \neq \mu_{i}$ for any $i$ implies $\left.V_{i}(\sigma) \cap M\right|_{N}=\langle 0\rangle$. Thus $\left.V_{s}(\sigma) \subseteq(X / M)\right|_{N}$, so that $\sigma$ is the npmv of irreducible $X / M$. The proposition follows.

The following result is partially contained in [2].

Corollary 4.7. There is one block of defect 1 for each coset of char $\left(H / \mathscr{C}_{H}(P)\right)$ as in Proposition 4.6, and for each $\lambda \in$ char $H$ there is exactly one irreducible $K G$ module $L$ with npmv $\lambda$.

Proof. This follows from the fact that there are $\left|\mathscr{C}_{H}(P)\right|$ blocks of full defect (since $H$ is abelian), Proposition 4.6 and (4.3).

Proposition 4.8. Every modular irreducible in a block $B$ of defect 1 may be written in the prime subfield $F$ of $K$ if and only if one of them may be so written.

Proof. Let $L$ be a modular irreducible in $B$ with $L \leftrightarrow V_{t}(\lambda)$. $L$ may be written in $F$ if and only if $L^{T}=L$ for all automorphisms $T$ of $K$ if and only if $V_{t}(\lambda)^{T}=V_{t}(\lambda)$ if and only if $\lambda^{T}=\lambda$ for all $T \in$ Aut $(K)$. Since $\alpha(H) \subseteq F, \lambda^{T}=\lambda$ if and only if $\left(\lambda \alpha^{i}\right)^{T}=\lambda \alpha^{i}$ for all integers $i$. Apply Proposition 4.6.

Remarks. (1) Tuan's result [15] that every modular irreducible in $B_{0}$, the principal block, can be written in $F$, follows immediately. (2) If $|H| \mid p-1$, then $\lambda(H) \subseteq F$ for all $\lambda \in$ char $H$ and in this case all modular irreducibles in blocks of defect 1 are written in $F$. 
Proposition 4.9. If $\mathscr{C}_{H}(P)$ is cyclic, there are at most two blocks containing a real stem.

Proof. If $B$ has a real stem, then $B$ contains a modular irreducible $L \leftrightarrow V_{d}(\lambda)$ and its dual $L^{*} \leftrightarrow V_{d}\left(\lambda^{-1} \alpha^{d-1}\right) . \lambda^{-1} \alpha^{d-1}=\lambda \alpha^{k}$ for some integer $k$ by Proposition 4.6. Thus $\left.\lambda^{2}\right|_{\mathscr{C}_{H}(P)}=1$. But if $\mathscr{C}_{H}(P)$ is cyclic then $\left.\lambda^{2}\right|_{\mathscr{C}_{H}(P)}=1$ if and only if $\lambda$ is in one of at most two fixed cosets of char $\left(H / \mathscr{C}_{H}(P)\right)$ in char $H$. Apply Corollary 4.7.

Remark. One of these blocks will be $B_{0}$, the principal block. Denote the other, if it exists, by $B_{2}$. $B_{2}$ may have a real stem consisting of a single vertex only.

The local theory easily yields the well-known

Proposition 4.10. If $L$ is a nonprojective indecomposable $K G$-module and $T \in$ Aut $K$, then $\left(L^{T}\right)^{*} \approx\left(L^{*}\right)^{T}$.

Proof. Say $L \leftrightarrow V_{d}(\lambda)$. Then

$$
\left(L^{T}\right)^{*} \leftrightarrow V_{d}\left(\lambda^{T}\right)^{*}=V_{d}\left(\left(\lambda^{T}\right)^{-1} \alpha^{d-1}\right)=V_{d}\left(\left(\lambda^{-1} \alpha^{d-1}\right)^{T}\right) \leftrightarrow\left(L^{*}\right)^{T} .
$$

$\S 4$ is concluded with a proposition of a general nature. The easy proof is omitted.

Proposition 4.11. Let $L$ be an indecomposable but not irreducible KG-module with $L \approx L^{*}$ and socle $L=W_{1}+W_{2}+\cdots+W_{t}$. Then socle $L \subseteq$ radical $L$ and $W_{1}^{*}$, $W_{2}^{*}, \ldots, W_{t}^{*}$ are the constituents of $L / \operatorname{rad} L$.

5. Lower bounds. We assume for the rest of this paper that group $G$ and module $L$ satisfy the hypotheses of Theorem 1 with $p \geqq 13$ and $\operatorname{dim}_{K} L=d<p$. Let $T=\bigcap_{n} G^{(n)}$, the intersection of the derived groups. Since $G$ is not of type $L_{2}(p), T$ and $\left.L\right|_{T}$ also satisfy Theorem 1 . Thus with no loss we assume $G=G^{\prime}$.

If $X$ is a $K G$-module such that $\left.X\right|_{P}$ is indecomposable, either $X$ is the trivial onedimensional module or $\operatorname{dim} X \geqq(7 / 10) p-\frac{1}{2} . N=P H$, where $H$ is an abelian $p^{\prime}-$ group, so $\S 2$ applies: $\left.L\right|_{N}=V_{d}(\lambda)$ for some $\lambda \in \operatorname{char}(H)$.

We use the following notation:

$$
\begin{aligned}
& s=p-d ; \\
& e=|N: C|=(p-1) / t ; \\
& Z=\mathscr{Z}(G) \text { and } z=|Z| .
\end{aligned}
$$

If $X$ is an indecomposable $K G$-module, write $X=X(u, \gamma)$ if and only if $X \leftrightarrow V_{u}(\gamma)$ by the Green correspondence.

$1_{0}=$ the trivial one-dimensional $K G$-module.

Proposition 5.1. $Z$ is cyclic and $z \mid$ d.

Proof. If $y \in Z, y$ acts on $L$ as the $d \times d$ scalar matrix $(\lambda(y))$. Thus $L$ faithful implies $\lambda$ is faithful on $Z$. Then $\lambda(Z) \subseteq K$ implies $Z$ is cyclic. Det $(\lambda(y))=\lambda^{d}(y)=1$ since $G=G^{\prime}$. Hence $z \mid d$. 
Let $M=M(d, \gamma)$ be another (not necessarily distinct) $K G$-module with $\operatorname{dim} M$ $=\operatorname{dim} L$ (in the sequel, $M$ is usually $L$ or $\left.L^{*}\right) .\left.M\right|_{N}=V_{d}(\gamma)$, so Lemma 2.6 implies

$$
\left.(L \otimes M)\right|_{N}=\sum_{i=0}^{s-1} V_{2 i+1}\left(\lambda \gamma \alpha^{s+i}\right)+\sum_{i=s}^{p-s-1} V_{p}\left(\lambda \gamma \alpha^{s+i}\right)
$$

Then by the Green correspondence, $L \otimes M=\sum_{i=0}^{s-1} L\left(2 i+1, \lambda \gamma \alpha^{s+i}\right)+Q$, where $Q$ is projective. By (5.2), for each integer $i$ with $0 \leqq i \leqq s-1$, we may choose a set of integers $\mathscr{S}_{i}$ such that $\mathscr{S}_{i} \cap \mathscr{S}_{k}=\varnothing$ if $i \neq k, \bigcup_{i=0}^{s-1} \mathscr{S}_{i}$ is contained in the set of integers $j$ such that $s \leqq j \leqq p-s-1$, and

$$
\left.L\left(2 i+1, \lambda \gamma \alpha^{s+i}\right)\right|_{N}=V_{2 i+1}\left(\lambda \gamma \alpha^{s+i}\right)+\sum_{j \in \mathscr{S}_{i}} V_{p}\left(\lambda \gamma \alpha^{s+\jmath}\right) .
$$

Let $m_{i}=\left|\mathscr{S}_{i}\right|$. Of course, $\mathscr{S}_{i}$ and $m_{i}$ are also functions of $\lambda, \gamma$, and $s$. We have

$$
\operatorname{dim} L\left(2 i+1, \lambda \gamma \alpha^{s+i}\right)=2 i+1+m_{i} p, \quad \text { and } \quad \sum_{i=0}^{s-1} m_{i} \leqq p-2 s .
$$

$m_{i}>0$ for $1 \leqq i \leqq s-1$, since $2 i+1<(7 / 10) p-\frac{1}{2}$, and $m_{0}=0$ if and only if $\lambda \gamma \alpha^{s}=\alpha^{0}$ if and only if $\gamma=\lambda^{-1} \alpha^{-s}$, which says $M \approx L^{*}$.

Using Lemma 2.3 and the assumption $G=G^{\prime}$, we have, for $0 \leqq i \leqq s-1$,

$$
\begin{aligned}
& 1=\operatorname{det} L\left(2 i+1, \lambda \gamma \alpha^{s+i}\right) \text { on } H \\
& =\left(\lambda \gamma \alpha^{s}\right)^{2 i+1} \prod_{j \in \mathscr{S}_{1}}\left(\lambda \gamma \alpha^{s}\right)^{p} \alpha^{j} \alpha^{-(p-1) / 2} \\
& =\left(\lambda \gamma \alpha^{s}\right)^{2 i+1+m_{i} p} \alpha^{-m_{i}(p-1) / 2} \prod_{j \in \mathscr{S}_{i}} \alpha^{j} .
\end{aligned}
$$

Now $L\left(2 i+1, \lambda \gamma \alpha^{s+i}\right) \approx L\left(2 i+1, \lambda \gamma \alpha^{s+i}\right)^{*}$ if and only if $\left(\lambda \gamma \alpha^{s+i}\right)^{2}=\alpha^{2 i}$ (by Lemma 2.3) if and only if $\left(\lambda \gamma \alpha^{s}\right)^{2}=1$. Thus either none or all of the nonprojective indecomposable summands of $L \otimes M$ are self-dual.

LEMMA 5.5. Suppose the nonprojective indecomposable summands of $L \otimes M$ are self-dual. The number of summands $L\left(2 i+1, \lambda \gamma \alpha^{s+i}\right)$ of $\operatorname{dim} 2 i+1+m_{i} p$ with $m_{i}$ odd is less than or equal to

$$
\begin{array}{ll}
t-1 & \text { if } t \text { is even, } \\
t & \text { if } t \text { is odd, } \\
t-2 & \text { if } t \text { is odd but } s>e / 2
\end{array}
$$

Proof. If $V_{p}(\mu)$ is a summand of $\left.L\left(2 i+1, \lambda \gamma \alpha^{s+i}\right)\right|_{N}$, so is $V_{p}(\mu)^{*}=V_{p}\left(\mu^{-1}\right)$. $\mu=\lambda \gamma \alpha^{s+j}$ implies $\mu^{-1}=\lambda \gamma \alpha^{s-j}$. Then for any $i$ with $0 \leqq i \leqq s-1,(5.4)$ implies

$$
1=\left(\lambda \gamma \alpha^{s}\right)^{2 i+1+m_{i} p \alpha^{-m_{i}(p-1) / 2}} \prod_{j \in \mathscr{S}_{i} ; 2 j \equiv 0(\bmod e)} \alpha^{j} .
$$


If $m_{i}$ is odd, then $2 i+1+m_{i} p$ even gives $\left(\lambda \gamma \alpha^{s}\right)^{2 i+1+m_{i} p}=1$, so that

$$
\alpha^{m_{i}(p-1) / 2}=\alpha^{(p-1) / 2}=\prod_{j \in \mathscr{S}_{i ;} 2 j \equiv 0(\bmod e)} \alpha^{j} .
$$

There is an odd number of such $j$. If $t$ is even, then $(p-1) / 2 \equiv 0(\bmod e)$ and there is an odd number of $j \equiv 0(\bmod e)$ in $\mathscr{S}_{i}$. If $t$ is odd, then $(p-1) / 2 \equiv e / 2(\bmod e)$, and there is an odd number of $j \equiv e / 2(\bmod e)$ in $\mathscr{S}_{i}$. (5.2) establishes the lemma.

THEOREM 5.7. $d \geqq \max \{p-e, 3(p-1) / 4\}$.

Proof. If $d$ is taken to be minimal, $L$ may be assumed absolutely irreducible. $d<p$ implies $L$ is in a block of defect 1 . Theorem 1 says $d \geqq(7 / 10) p-\frac{1}{2}$, so by (4.3), $d \geqq p-e$. If $e \leqq(p-1) / 4$ then $d \geqq(3 p+1) / 4$. If $e=(p-1) / 3$ and $s \leqq e / 2$ then $d \geqq(5 p+1) / 6$. So we may assume $t \leqq 3$ and if $t=3$ then $s>e / 2$.

Let $M=L^{*}$. Then $\gamma=\lambda^{-1} \alpha^{-s}$, so $\lambda \gamma \alpha^{s}=1$. Lemma 5.5 implies at most one $m_{i}$ is odd. By (5.3),

$$
1+2(s-2) \leqq \sum_{i=1}^{s-1} m_{i} \leqq p-2 s
$$

whence $s \leqq(p+3) / 4$.

Proposition 5.8. If $z$ is odd then either $d>p-e$ or $e=2$.

Proof. By Theorem 5.7 we may assume $d=p-e$ and $L$ is absolutely irreducible. $e<p-1$ implies $\operatorname{sep} L=e$, so that $L$ lifts to an ordinary irreducible which is exceptional. Then a theorem of Feit [6] gives $e=2$.

Now let $M=L$. (5.4) gives, for $0 \leqq i \leqq s-1$,

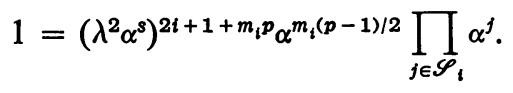

Since $\alpha$ is trivial on $Z,\left.\left(\lambda^{2}\right)^{2 i+1+m_{t} p}\right|_{Z}=1$. Since $L$ is faithful, $\lambda$ is faithful on $Z$, so that

$$
z \mid 2\left(2 i+1+m_{i} p\right), \quad 0 \leqq i \leqq s-1 .
$$

The next theorem shows that $d$ is bounded below at least as a function of the order of $Z$.

THEOREM 5.11. If $b \mid z$ with $b$ an odd integer, set $s=b q+r, q$ and $r$ integers with $0 \leqq r<b$. Then

$$
s \leqq(2 /(b+5))(p+r(b-r) / 2) .
$$

If $4 c \mid z$, set $s=c w+u, c, w$, and $u$ integers with $0 \leqq u<c$. Then all the $m_{i}$ (from $L \otimes L)$ are odd and

$$
s \leqq(1 /(c+2))(p+u(c-u))
$$


Proof. If odd $b \mid z$, then (5.10) implies $b \mid 2 i+1+m_{i} p, 0 \leqq i \leqq s-1$, so for any $0 \leqq i, j \leqq s-1, b \mid 2(i-j)+p\left(m_{i}-m_{j}\right)$. Hence if $|i-j|<b, b \nmid i-j$ so $b \nmid m_{i}-m_{j}$. In particular $m_{i} \neq m_{j}$. Thus

$$
\begin{aligned}
\sum_{b \text { consecutive integers } i} m_{i} & \geqq \sum_{j=1}^{b} j=b(b+1) / 2, \\
p-2 s & \geqq \sum_{i=0}^{s-1} m_{i} \geqq q b(b+1) / 2+r(r+1) / 2 \\
& =(s-r)(b+1) / 2+r(r+1) / 2 .
\end{aligned}
$$

Solving for $s$ proves the first statement.

If $4 c \mid z$, then $2 c \mid 2 i+1+m_{i} p, 0 \leqq i \leqq s-1$, by (5.10), so each $m_{i}$ is odd. $2 c \mid 2(i-j)+p\left(m_{i}-m_{j}\right)$ for $0 \leqq i, j \leqq s-1$. So if $|i-j|<c, c \nmid m_{i}-m_{j}$ and

$$
\begin{aligned}
\sum_{c \text { consecutive integers } i} m_{i} & \geqq \sum_{j=1}^{c} 2 j-1=c^{2}, \\
p-2 s & \geqq \sum_{i=0}^{s-1} m_{i} \geqq w c^{2}+u^{2}=(s-u) c+u^{2} .
\end{aligned}
$$

Solve for $s$ to complete the proof.

If $e=|\operatorname{char}(H / Z)|=|\langle\alpha\rangle|$ is even, then we may sensibly speak of the parity of an element of $\langle\alpha\rangle$ as an odd or even power of $\alpha$.

THEOREM 5.12. Assume $e$ is even. If either (i) $z$ is odd and $d$ is even, or (ii) $z=2$ and $\lambda^{2}$ is even, then $d=p-1$ and $p \equiv 1(\bmod 4)$. If $z=4$, then $d>(4 p) / 5$.

Proof. Lemma 3.3 implies that if $j \in \mathscr{S}_{i}$ for $M=L$ in $(5.2)$, then $j \equiv i(\bmod 2)$. If $(p-1) / 2$ is even and $i$ is odd, then $\alpha^{m_{i}(p-1) / 2}$ is even and $\prod_{j \in \mathscr{S}_{i}} \alpha^{j}$ has the same parity as $m_{i}$. If $(p-1) / 2$ is odd and $i$ is even, then $\prod_{j \in \mathscr{S}_{i}} \alpha^{j}$ is even and $\alpha^{m_{i}(p-1) / 2}$ has the same parity as $m_{i}$. Thus if $(p-1) / 2 \equiv i+1(\bmod 2), \alpha^{m_{i}(p-1) / 2} \prod_{j \in \mathscr{P}_{i}} \alpha^{j}$ has the same parity as $m_{i}$. Furthermore, $d$ is even under any of the hypotheses, so $\alpha^{s\left(2 i+1+m_{i} p\right)}$ has opposite parity from $m_{i}$. Then (5.9) implies $\left(\lambda^{2}\right)^{2 i+1+m_{i} p}$ is odd for all $0 \leqq i \leqq s-1$ with $i \equiv(p+1) / 2(\bmod 2)$.

$\lambda^{z} \in\langle\alpha\rangle$. By (5.10), $\left(\lambda^{2}\right)^{2 i+1+m_{i} p}$ is even for all $0 \leqq i \leqq s-1$ if either (i) or (ii) hold. Then in this event, $(p-1) / 2$ is even and $s=1$. The first statement is proved.

Suppose $4 \mid z$. Then all $m_{i}$ are odd, $0 \leqq i \leqq s-1$. If $i \equiv(p-1) / 2(\bmod 2)$, then $\alpha^{s\left(2 i+1+m_{i} p\right)} \alpha^{m_{i}(p-1) / 2} \prod_{j \in \mathscr{S}_{i}} \alpha^{j}$ is even, and hence so is $\left(\lambda^{2}\right)^{2 i+1+m_{i} p}$ by (5.9). Assume $d<p-1$. Then $\left(\lambda^{2}\right)^{2 i+1+m_{i} p}$ is odd for some $i \leqq s-1$. (5.10) implies $\lambda^{z}$ is odd. It follows that, for $0 \leqq i \leqq s-1$,

$$
2\left(2 i+1+m_{i} p\right) / z \equiv i+(p-1) / 2 \quad(\bmod 2) .
$$

If $z=4,2\left(2 i+1+m_{i} p\right) / z=\left(2 i+1+m_{i}+m_{i}(p-1)\right) / 2=\left(m_{i}+1\right) / 2+i+m_{i}(p-1) / 2 \equiv$ $\left(m_{i}+1\right) / 2+i+(p-1) / 2(\bmod 2)$. Thus $m_{i} \equiv 3(\bmod 4), 0 \leqq i \leqq s-1$. Then by (5.3), $3 s \leqq \sum_{i=0}^{s-1} m_{i} \leqq p-2 s$. This proves the second statement. 
REMARK 5.13. The nonprojective summands of $L \otimes L$ are self-dual if and only if $\left(\lambda^{2} \alpha^{s}\right)^{2}=1$. This implies $z \mid 4$.

THEOREM 5.14. If $z=4$ and $\left(\lambda^{2} \alpha^{3}\right)^{2}=1$ then

$$
\begin{aligned}
d \geqq p-1 \text { and } p \equiv 1(\bmod 4) & \text { if } e \text { is even, } \\
\geqq p-t+1 & \text { if } e \text { is odd. }
\end{aligned}
$$

Proof. All the $m_{i}$ (from $L \otimes L$ ), $0 \leqq i \leqq s-1$, are odd by Theorem 5.11. Then Lemma 5.5 implies $d \geqq p-t+1$ if $e$ is odd. The proof of Lemma 5.5 shows that for all $0 \leqq i \leqq s-1$, there is some $j \equiv(p-1) / 2(\bmod e)$ in $\mathscr{S}_{i}$. If $j \in \mathscr{S}_{i}$ then $j \equiv i(\bmod 2)$ by Lemma 3.3. If $e$ is even, then $(p-1) / 2 \equiv i(\bmod 2)$ for all $0 \leqq i \leqq s-1$. Hence $s=1$ and $(p-1) / 2$ is even.

LEMMA 5.15. Suppose $L \approx L^{*}$ and $M=M(d, \gamma) \approx M^{*} \not L L$ are in the same $p$-block with $\operatorname{dim} M=d$. Then

$$
\begin{aligned}
& s \leqq t-1 \text { if } t \text { is even, } \\
& \leqq t \quad \text { if } t \text { is odd, } \\
& \leqq t-2 \text { if } t \text { is odd and } s>e / 2 \text {. }
\end{aligned}
$$

Proof. $\gamma^{2}=\lambda^{2}=\alpha^{d-1}$, and by Proposition 4.6, $\gamma \lambda^{-1} \in\langle\alpha\rangle$. Hence $\gamma=\lambda \alpha^{e / 2}$ and $\lambda \gamma \alpha^{s}=\alpha^{e / 2}$. Thus the nonprojective summands of $L \otimes M$ are self-dual, and (5.6) implies, for all $0 \leqq i \leqq s-1$,

$$
\begin{aligned}
1 & =\left(\alpha^{e / 2}\right)^{2 i+1+m_{l} p} \alpha^{m_{i}(p-1) / 2} \prod_{j \in \mathscr{S}_{i} ; 2 j \equiv 0(\bmod e)} \alpha^{j} \\
& =\alpha^{e / 2+m_{i}(e / 2-(p-1) / 2)} \prod_{j \in \mathscr{S}_{i} ; 2 j \equiv 0(\bmod e)} \alpha^{j} .
\end{aligned}
$$

If $m_{i}$ is odd, then as in Lemma 5.5, $t$ even implies there is an odd number of $j \equiv 0$ $(\bmod e)$ in $\mathscr{S}_{i}$, and $t$ odd implies there is an odd number of $j \equiv e / 2(\bmod e)$ in $\mathscr{S}_{i}$. If $m_{i}$ is even, there is an even number of $j \in \mathscr{S}_{i}$ with $2 j \equiv 0(\bmod e)$, and (5.16) gives

$$
1=\alpha^{e / 2} \prod_{j \in \mathscr{S}_{i} ; 2 j \equiv 0(\bmod e)} \alpha^{j} .
$$

Hence there is an odd number of $j \equiv e / 2(\bmod e)$ in $\mathscr{S}_{i}$, and thus also an odd number of $j \equiv 0(\bmod e)$. Done by (5.2).

Lemma 5.17. Let $L$ be self-dual, $z=2$ and $e$ be even. Then $H$ is cyclic.

Proof. $H / Z$ is cyclic and $z=2$. Thus if $H$ is not cyclic, $H=E \times Z$ where $E \approx H / Z$ acts faithfully on $P$. Since $\left.L\right|_{N}=V_{d}(\lambda),\left.L\right|_{P E}=V_{d}\left(\left.\lambda\right|_{E}\right)=V_{d}\left(\alpha^{k}\right)$ for some integer $k$. $L \approx L^{*}$ implies $\lambda^{2}=\alpha^{d-1}$, whence $\left(\left.\lambda\right|_{E}\right)^{2}=\alpha^{2 k}=\alpha^{d-1}$. Since $e$ is even, $d-1$ must be even and $d$ is odd. But $2=z \mid d$, a contradiction. 
THEOREM 5.18. Let $L$ be self-dual, $z=2$ (so that $L \in B_{2}$ ) and $e=(p-1) / t$ where $t$ is odd. Then $L$ has an algebraic conjugate in $B_{2}$ and $d \geqq p-t$.

Proof. Let $Q$ be the Sylow 2-subgroup of $H$. Since $|H|=z(p-1) / t$, where $z=2$ and $t$ is odd, $\nu_{2}(|Q|)=\nu_{2}(p-1)+1$. By the above lemma, $H$, and hence $Q$, is cyclic. Thus, $\lambda$ faithful on $Z$ implies $\lambda$ is faithful on $Q$, so $\lambda(Q) \nsubseteq F$, the prime subfield of $K$. Then there exists $T \in$ Aut $(K)$ with $\lambda^{T} \neq \lambda$, and hence $L^{T}=L\left(d, \lambda^{T}\right) \not 2 L$. Since $\left(\lambda^{T}\right)^{2}=\left(\lambda^{2}\right)^{T}=\alpha^{d-1},\left(L^{T}\right)^{*} \approx L^{T}$. $T$ preserves $B_{0}$, hence $L$ and $L^{T} \in B_{2}$ by Proposition 4.9. Lemma 5.15 implies $d \geqq p-t$.

6. A minimal case. After dispensing with some elementary facts, we extract further information when $L$ has the smallest degree allowed by Theorem 5.7, that is, $3(p-1) / 4$.

Proposition 6.1. If $d<p-1$, then $L$ is irreducible.

Proof. $\left.L\right|_{P}=V_{d}$ has a unique one-dimensional space of invariants, so the socle of $L$ is irreducible and every submodule of $L$ is indecomposable. If $1_{0}$ occurs twice consecutively in a composition series for $L$, then Proposition 4.5 implies $1=1 \alpha^{-1}$, so $e=1$, a contradiction. Then $L$ has a unique nontrivial constituent $R \leftrightarrow V_{r}(\rho)$. If $L$ has composition series $1_{0}, R, 1_{0}$ then $\lambda=1$ and $1=1 \alpha^{-(r+1)}$ by Proposition 4.5. Hence $d=r+2 \equiv 1(\bmod e)$, so $d>r \geqq p-e$ implies $d=p$, a contradiction.

Thus $L$ has composition series either $1_{0}, R$ or $R, 1_{0}$. Replacing $L$ by $L^{*}$ if necessary, we may assume the former. Then $\lambda=1, \rho=\alpha^{-1}$, and $R$ is a constituent of $Q_{0}$, the projective indecomposable with socle $1_{0} . R$ is adjacent to $1_{0}$ in the graph of $B_{0}$. If $R \not R^{*}$ then sep $R=r$ and $R, R^{*}$ and $1_{0}$ separate $2 r+1$ vertices from the exceptional. Hence, $p-1 \geqq e \geqq 2 r+1 \geqq 3(p-1) / 2+1$, a contradiction. If $R \approx R^{*}$ then $\alpha^{-2}=\rho^{2}=\alpha^{r-1}$. Hence $r \equiv-1(\bmod e)$. But $r \geqq p-e$ gives $r=p-2$ and $d=p-1$.

Proposition 6.2. $\lambda^{2} \alpha^{s}=\alpha^{c}$ for some integer $c$ with $|c| \leqq s-1$ if and only if $c=0$ (i.e., $L \approx L^{*}$ ).

Proof. $L \approx L^{*}$ if and only if there are invariants in $\left(L^{*}\right)^{*} \otimes L=L \otimes L$ (and equivalently in $\left.L^{*} \otimes L^{*}\right)$, since $L$ is irreducible for $d<p-1$. This follows from there being invariants in $\sum_{i=0}^{s-1} V_{2 i+1}\left(\lambda^{2} \alpha^{s+i}\right)$ or $\sum_{i=0}^{s-1} V_{2 i+1}\left(\lambda^{-2} \alpha^{-s+i}\right)$ by Theorem 4.1. This says $\lambda^{2} \alpha^{s}=\alpha^{c}$ for some $c$ with $|c| \leqq s-1$. When $L \approx L^{*}, \lambda^{2} \alpha^{s}=1$, and $\alpha^{c}=1$ for some $c$ with $|c| \leqq s-1$ if and only if $c=0$, since $s \leqq e$.

LemMA 6.3. Suppose $z \mid 2$ and $\left(\lambda^{2} \alpha^{s}\right)^{2} \neq 1$, so that (replacing $L$ by $L^{*}$ if necessary) $\lambda^{2} \alpha^{s}=\alpha^{k}$ with $e / 2<k<e$. If there exist integers $0 \leqq b, c<s$ such that $k+b+c \geqq e$ and $|b-c| \leqq e-k$ then $\operatorname{Hom}_{K G}\left(L\left(2 c+1, \alpha^{c}\right), L\left(2 b+1, \lambda^{2} \alpha^{s+b}\right)\right) \neq 0$.

Proof. If one of $b$ or $c$ is less than $(p-1) / 4$ then $2(b+c)+2 \leqq p$, so by Lemma 2.4 $V_{2 c+1}\left(\alpha^{c}\right) \otimes V_{2 b+1}\left(\lambda^{2} \alpha^{s+b}\right)$ has main $H$-values $\alpha^{k+b+c}, \alpha^{k+b+c+1}, \ldots, \alpha^{k+|b-c|}$ and hence has invariants. If $b=c=(p-1) / 4$ then $s=(p+3) / 4$ implies $k \geqq e / 2+1$ (since $e \geqq(p-1) / 3$ and $4 \mid p-1$ says $e$ is even), so $V_{2 c+1}\left(\alpha^{c}\right) \otimes V_{2 b+1}\left(\lambda^{2} \alpha^{s+b}\right)$ has npmv's 
$\alpha^{k+(p-1) / 2} \alpha^{-((p-1) / 2+1)+1+i}=\alpha^{k+i}, 0 \leqq i \leqq(p-1) / 2-1$, by Lemma 2.6. So in either case, there are invariants in $L\left(2 c+1, \alpha^{c}\right)^{*} \otimes L\left(2 b+1, \lambda^{2} \alpha^{s+b}\right)$ by Theorem 4.1.

TheOREM 6.4. If $d=3(p-1) / 4$ then $L \approx L^{*}, z=2$, and $e=(p-1) / 2$.

Proof. Let $L_{i}=L\left(2 i+1, \alpha^{i}\right)$ and $N_{i}=L\left(2 i+1, \lambda^{2} \alpha^{s+i}\right)$ for $0 \leqq i \leqq s-1$. These are the nonprojective summands of $L \otimes L^{*}$ and $L \otimes L$, respectively. Set $\operatorname{dim} L_{i}$ $=2 i+1+m_{i} p$ and $\operatorname{dim} N_{i}=2 i+1+n_{i} p$.

$L$ is irreducible. By Theorem 5.7 and its proof we may assume $t \leqq 3$, one $m_{i}=1$ and all the others are 2 for $1 \leqq i \leqq s-1$. By Theorem $5.11, z \mid 4$. Since $4 \mid p-1$, $e$ is even. Then Theorem 5.12 implies $z \mid 2$.

First, suppose $L \not L^{*}$.

(i) Suppose $\left(\lambda^{2} \alpha^{s}\right)^{2}=1$. Since $t \leqq 3$, and $t=3$ implies $s>e / 2$, Lemma 5.5 says the number of odd $n_{i}$ is less than or equal to 1 . Then

$$
2(s-1)+1 \leqq \sum_{i=0}^{s-1} n_{i} \leqq p-2 s,
$$

so $s \leqq(p+1) / 4$, a contradiction.

(ii) Suppose $\left(\lambda^{2} \alpha^{s}\right)^{2} \neq 1 . z \mid 2$ implies $L, L^{*}$ are both in the same block $B$ by Corollary 4.7. $B$ must have a real stem, and $L, L^{*}$ separate a total of $2 s=(p+3) / 2$ vertices from the exceptional. So $e=p-1$. $\lambda^{2} \alpha^{s}=\alpha^{k}$ where $e / 2+1 \leqq k<e$. Then $k+(s-1)+(s-2)=k+(p-1) / 2-1 \geqq e$, so Lemma 6.3 implies there exist nonzero $K G$-homomorphisms from $L_{s-1}$ to $N_{s-1}$ and to $N_{s-2}$.

Since all $m_{i} \leqq 2, \operatorname{dim} L_{s-1} \leqq 2 p+2 s-1=2 p+(p+1) / 2<4(3 / 4)(p-1)$. Hence $L_{s-1}$ has at most three nontrivial irreducible constituents. Since $L_{s-1}$ has no invariants, by Theorem 4.1, and is self-dual, Proposition 4.11 implies $L_{s-1}$ has a unique minimal submodule $W \neq 1_{0}$ and a unique maximal submodule $M$ with $\operatorname{dim} M$ $=a p+m, 0<m<p . W^{*}=L_{s-1} / M$, so if $W^{*}$ has a pmv, then it is a mv of any nonzero $K G$-homomorphic image of $L_{s-1}$, hence of $N_{s-1}$ and $N_{s-2}$. But the mv's of all the $N_{i}$ are distinct when $e=p-1$, by (5.2), a contradiction. Thus $3(p-1) / 4$ $\leqq \operatorname{dim} W=w<p$, and $W$ has a unique $\operatorname{mv} \gamma$. Let $\gamma^{*}=\gamma^{-1} \alpha^{w-1}$, the mv of $W^{*}$. Then $\gamma^{*}$ is not a mv of some $N_{u}, u=s-1$ or $s-2$. Since $(p+1) / 2=2 s-1<w$, $m+w>p$, so Proposition 4.5 implies $\gamma^{*}=\alpha^{s-1}$, the npmv of $L_{s-1}$.

Let $S$ be the kernel of the homomorphism $L_{s-1} \rightarrow N_{u}$. Then $W^{*}$ is not a submodule of $L_{s-1} / S, S \neq\{0\}$, and $N_{u}$ has no invariants, so $L_{s-1} / S$ has a unique minimal submodule $R$, where $R \approx R^{*}$ is the third nontrivial constituent of $L_{s-1}$. Thus $L_{s-1} / S$ has composition series $R, W^{*}$ or $R, 1_{0}, W^{*}$ and each submodule of $L_{s-1} / S$ is indecomposable. $\gamma^{*}$ cannot be a mv of $L_{s-1} / S$, so rem $R+\operatorname{dim} W<p$ by Proposition 4.5. Then $\operatorname{dim} R>p$. But $\operatorname{dim} R \leqq(5 p+1) / 2-3(p-1) / 2=p+2$.

Since $L_{s-1}$ has unique minimal submodule $W, L_{s-1}$ is properly contained in the projective indecomposable with socle $W$. Then all constituents of $L_{s-1}$ lie on edges 
adjacent to $W$ in the tree of $B_{0}$, and $e=p-1$ implies none occurs more than once in $L_{s-1}$. Thus $W \not W^{*}$ and on the graph

$$
\begin{array}{l|l}
W & R \\
W^{*} &
\end{array}
$$

Take the vertex pictured as the exceptional. $\operatorname{dim} W \geqq 3(p-1) / 4$ implies sep $W$ $=p$-rem $W$. Then sep $R=p$-rem $R \geqq p-2$, a contradiction.

Second, suppose $L \approx L^{*}$.

Let $L \otimes L=A+B$, the symmetric and skew decomposition. Lemma 3.3 gives $\sum_{i \equiv s(\bmod 2)} L_{i} \subseteq A, \sum_{i \equiv s-1(\bmod 2)} L_{i} \subseteq B$.

If $z=1, L \in B_{0} . e$ is even, so $s$ is even by Theorem 5.12. Then

$$
\sum_{i \equiv s-1(\bmod 2)} m_{i}=m_{1}+m_{3}+\cdots+m_{s-1} \leqq(p-2 s-1) / 2
$$

by Lemma 3.3. Hence $2(s / 2)-1 \leqq(p-2 s-1) / 2$, since all but one $m_{i}=2$. This gives $s \leqq(p+1) / 4$, a contradiction.

It follows that $z=2$. Theorem 5.18 implies $e=(p-1) / 2$.

Actually, a good deal more is known in this situation. Each of the $L_{i}$ (except perhaps $\left.L_{1}\right)$ is irreducible, and the degree of the exceptional characters in $B_{0}$ is either $(3 p+1) / 2$ or $(5 p+1) / 2([0$, Theorems $9.3,9.4]$ and other unpublished results of the author). Of course, the existence of such a group is not at all certain.

7. Functions of $|N: C|$.

THEOREM 7.1.

$$
\begin{aligned}
d & \geqq p-(e / 2+1) & & \text { (e even) } \\
& \geqq p-((e-1) / 2+t) & & \text { (e odd }) .
\end{aligned}
$$

Proof. We may assume $e<p-1$. Let $B^{\prime}$ be the block in which lie all the nonprojective indecomposable summands of $L \otimes L$. We denote these by

$$
N_{i}=L\left(2 i+1, \lambda^{2} \alpha^{s+i}\right), \quad 0 \leqq i \leqq s-1 .
$$

Let $\chi$ be an exceptional ordinary irreducible character in $B^{\prime} . \chi(1) \equiv \varepsilon e(\bmod p)$, where $\varepsilon= \pm 1$. Thompson [14, Theorem 1] has shown that there is an $\mathcal{O}$-free $\mathcal{O} G$ module $X$ affording $\chi$ such that $W=X \mid \mathscr{P} X$ has irreducible socle. If $M$ is an irreducible $K G$-module which is a constituent of $W$, then $M$ appears just once in any composition series for $W$. If $\varepsilon=1$, then Rothschild's argument shows $\sum$ rem $M$ $=e$, where the sum is taken over all constituents of $W$. If $\varepsilon=-1$, then rem $M$ $=p$-sep $M$ and $\sum \operatorname{sep} M=e$. So if we sum over any subset containing, say, $n$ of the constituents of $W$,

$$
n p>\sum \operatorname{rem} M>(n-1) p .
$$

A result of Janusz [12, Theorem 7.1] implies that $W$ is uniserial. (This can also be proved directly, using Proposition 4.5.) 
Let $Y=W / \operatorname{rad} W . Y$ is irreducible. Let $R$ be any nonzero $K G$-homomorphic image of $W$. Then $Y \approx R / \operatorname{rad} R$. Let $S=\operatorname{rad} W / \operatorname{rad}(\operatorname{rad} W)$.

If $\varepsilon=1$, let $Y \leftrightarrow V_{p-y}(\gamma)$, where $y=\operatorname{sep} Y$. By (7.2) and Proposition 4.5, $\gamma$ is the unique npmv of each of the $R$.

If $\varepsilon=-1$, either $Y$ has a pmv $\tau$ or $Y=1_{0}$. If the former is true, then $\tau$ is a pmv of each $R$. If the latter case holds then $0 \neq S \neq 1_{0}$ and $S$ has a pmv $\sigma$. Then either $R \approx 1_{0}$ or $R$ has $S$ as a constituent and $\sigma$ as a pmv.

We conclude that all modules which are nonzero $K G$-homomorphic images of $W$ and which are not equal to $1_{0}$ have a main value in common.

Suppose $s \geqq(e+1) / 2$. $W$ has Green correspondent either $V_{e}\left(\lambda^{2} \alpha^{k}\right)$ if $\varepsilon=1$, or $V_{p-e}\left(\lambda^{2} \alpha^{k}\right)$ if $\varepsilon=-1$, for some integer $k$. For each $i$ with $(e-1) / 2 \leqq i<s$, the npmv's of $V_{e}\left(\lambda^{2} \alpha^{k}\right)^{*} \otimes V_{2 i+1}\left(\lambda^{2} \alpha^{s+i}\right)$ are $\alpha^{-k-1+s+i-j}, 0 \leqq j \leqq e-1$, by Lemma 2.4. The npmv's of $V_{p-e}\left(\lambda^{2} \alpha^{k}\right)^{*} \otimes V_{2 i+1}\left(\lambda^{2} \alpha^{s+i}\right)$ are $\alpha^{-k+s-i+j}, 0 \leqq j \leqq e-1$, by Lemma 2.6. Since $|\langle\alpha\rangle|=e$, in either case $W^{*} \otimes N_{i}$ has npmv $\alpha^{0}$. By Theorem 4.1, there exists a nonzero $K G$-homomorphism from $W$ into $N_{i}$, for all $(e-1) / 2 \leqq i<s$. Since no such $N_{i}$ has invariants, the homomorphic image is never $1_{0}$. It follows that all the $N_{i}$ with $(e-1) / 2 \leqq i<s$ have a main value in common.

Suppose $e$ is even. Then Lemma 3.3 implies for $0 \leqq i, j<s, N_{i}$ and $N_{j}$ have no main values in common unless $i \equiv j(\bmod 2)$. Thus if $s>e / 2+1$, the existence of $N_{e / 2}$ and $N_{(e / 2)+1}$ as summands of $L \otimes L$ forces a contradiction. So if $e$ is even, $d \geqq p-(e / 2+1)$.

Suppose $e$ is odd. By (5.2) for $L \otimes L$, a given $\gamma \in$ char $H$ can be a npmv of at most one $N_{i}$, and a pmv of at most $t-1$ of the $N_{i}, 0 \leqq i \leqq s-1$. Since all the $N_{i}$ with $(e-1) / 2 \leqq i \leqq s-1$ have a main value in common, it follows that $t \leqq s-(e-1) / 2$. Therefore $d \geqq p-((e-1) / 2+t)$.

Remarks. Let $G$ be a finite group with a Sylow $p$-subgroup $P$ of prime order $p$. Assume that $P$ is not a normal subgroup of $G$, and that $G$ has a faithful irreducible complex representation of degree $n<p-1$. Then if $p>7$, either $G / Z \approx P S L(2, p)$ with $n=(p \pm 1) / 2$, or $G$ satisfies the hypotheses of Theorem 1 , with $d=n=p-e$ and $t=(p-1) / e \geqq 3[2, \mathrm{II}],[3],[6],[15]$. Assume the latter possibility. If $d<p-2$, then $z$ is even [6, Theorem 1]. In particular, $e$ must be odd and $t$ even, which also follows from Theorem 7.1 above. Theorem 7.1 also shows $p-e \geqq p-((e-1) / 2+t)$ which gives $p \leqq 2 t^{2}-t+1$. This improves Brauer's inequality $p \leqq t^{3}-t+1$ [3]. We will show in a separate paper that in fact $p \leqq t^{2}-3 t+1$.

8. Small primes. The results and methods of this paper have been applied to primes $p, 13 \leqq p \leqq 31$, to eliminate some possibilities for $d<p-2$, where $L=L(d, \lambda)$ satisfies Theorem 1 and $G=G^{\prime}$. The chart below lists all cases remaining open. From over 300 numerical possibilities for $e \mid p-1, d \geqq \max \{3(p-1) / 4, p-e\}$, and $z \mid d$, exactly 98 still remain. Work in progress may soon eliminate more cases. On the other hand, some new groups may arise. 
112

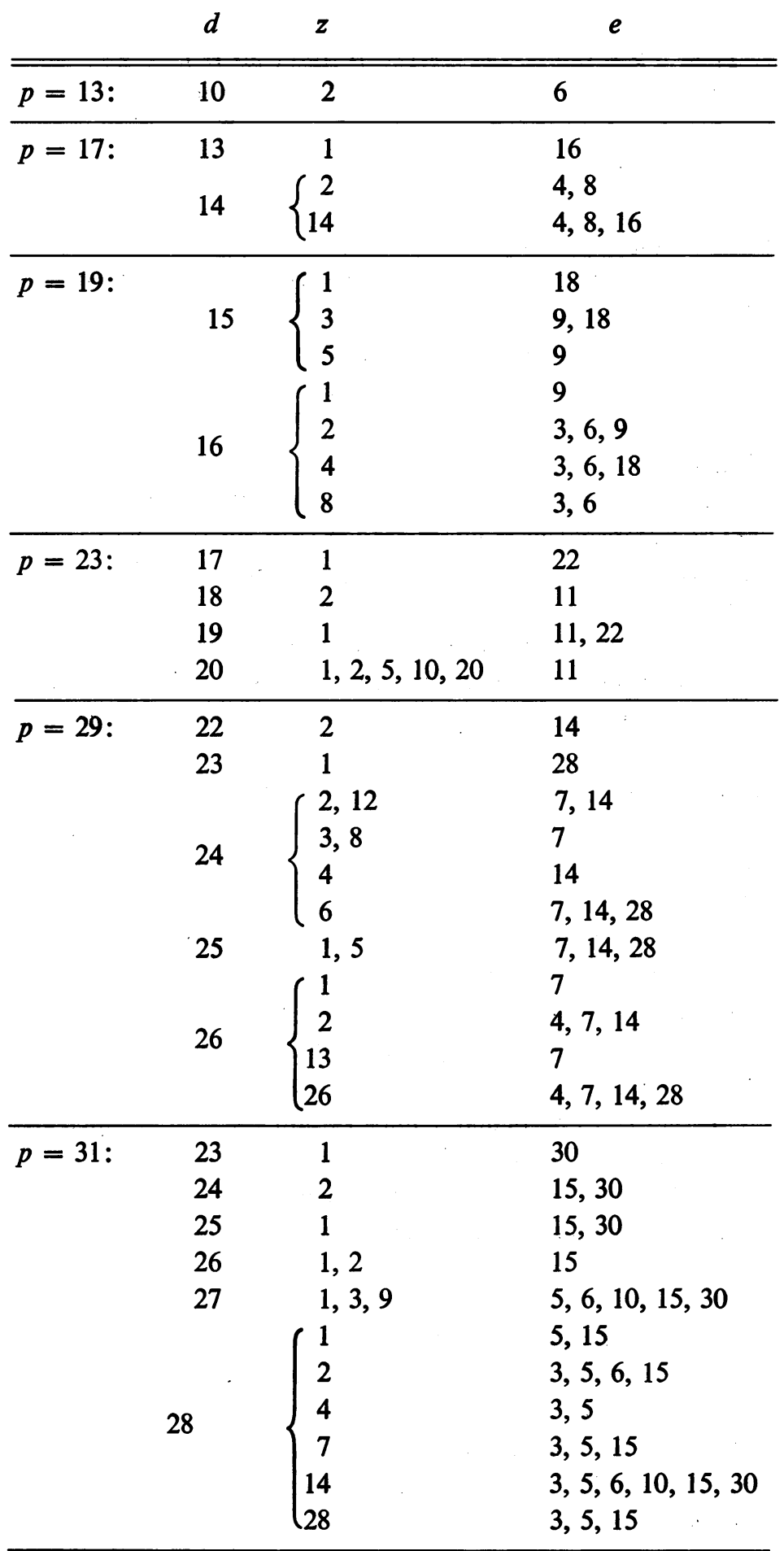




\section{REFERENCES}

0. H. Blau, On the degree of some finite linear groups, Ph.D. Thesis, Yale University, New Haven, Conn., 1969.

1. R. Brauer, Investigations on group characters, Ann. of Math. (2) 42 (1941), 936-958. MR 3, 196.

2. - On groups whose order contains a prime number to the first power. I, II, Amer. J. Math. 64 (1942), 421-440. MR 4, 1; MR 4, 2.

3. - Some results on finite groups whose order contains a prime to the first power, Nagoya Math. J. 27 (1966), 381-399. MR 33 \#7402.

4. E. C. Dade, Blocks with cyclic defect groups, Ann. of Math. (2) 84 (1966), 20-48. MR 34 \#251.

5. W. Feit, Groups with a cyclic Sylow subgroup, Nagoya Math. J. 27 (1966), 571-584. MR 33 \#7404.

6. - On finite linear groups, J. Algebra 5 (1967), 378-400. MR 34 \#7632.

7. - Modular representations of finite groups, Lecture Notes, Yale University, New Haven, Conn., 1969.

8. J. A. Green, On the indecomposable representations of a finite group, Math. Z. 70 (1958/59), 430-445. MR 24 \#A1304.

9. - Blocks of modular representations, Math. Z. 79 (1962), 100-115. MR 25 \#5114.

10. — The modular representation algebra of a finite group, Illinois J. Math. 6 (1962), 607-619. MR 25 \#5106.

11. Z. Janko, A new finite simple group with abelian Sylow 2-subgroups and its characterization, J. Algebra 3 (1966), 147-186. MR 33 \#1359.

12. G. J. Janusz, Indecomposable modules for finite groups, Ann. of Math. (2) 89 (1969), 209-241. MR 39 \#5622.

13. B. Rothschild, Degrees of irreducible modular characters of blocks with cyclic defect groups, Bull. Amer. Math. Soc. 73 (1967), 102-104. MR 34 \#4381.

14. J. G. Thompson, Vertices and sources, J. Algebra 6 (1967), 1-6. MR 34 \#7677.

15. H. F. Tuan, On groups whose orders contain a prime number to the first power, Ann. of Math. (2) 45 (1944), 110-140. MR 5, 143.

NORTHERN ILLINOIS UNIVERSITY,

De Kalb, IllinoIs 60115 